\title{
Erythrocyte Adenylate Kinase Isoenzyme as a Marker for Hemolysis George Thomas ${ }^{1}$ and Vadiraja V. Murthy* Department of Pathology, Albert Einstein College of Medicine, Bronx, New York
}

The presence in serum of adenylate kinase isoenzyme originating from erythrocyte can be useful as a marker for detecting hemolysis. We have presented preliminary evidence for identifying hemolytic anemia patients earlier by determining erythrocyte AK isoenzyme activity in serum (or plasma) rather than using measurement of plasma hemoglobin concentration. This test being quite specific for hemolysis should find use as a quick method for estimating the extent of in vivo hemolysis in hemolytic patients earlier than heretofore possible. J. Clin. Lab.Anal. 11:351-356, $1997 . \quad$ (c) 1997 Wiley-Liss, Inc.

Key words: adenylate kinase; erythrocyte specific isoenzyme; hemolytic anemia; enzyme marker for hemolysis

\section{INTRODUCTION}

Adenylate kinase activity in serum mostly consists of myokinase or muscle adenylate kinase isoenzyme and occasionally adenylate kinase isoenzyme from erythrocytes after in vivo or in vitro damage to the integrity of their membrane structure. This isoenzyme originating from red blood cells is the predominant AK isoenzyme fraction present in hemolyzed serum. We have recently shown that erythrocyte AK can be fractionated by electrophoresis and that it migrates on agarose gel in manner similar to the MM isoenzyme of creatine kinase (CK) in serum (1). This observation led us to evaluate the utility of this approach to detect in vivo hemolysis based on erythrocyte AK isoenzyme activity present in serum. To our knowledge, this is the first demonstration of the use of erythrocyte specific AK isoenzyme activity for assessing in vivo hemolysis in hospitalized anemic patients.

\section{MATERIALS AND METHODS}

We used the Helena REP CK electrophoresis apparatus to fractionate the erythrocyte AK isoenzyme. Agarose gels used for fractionating CK isoenzymes were purchased from Helena Laboratories, Beaumont, TX. All other reagents were purchased from Sigma, St. Louis, MO.

\section{Assays}

The reagent used for visualizing AK activity on the gel contained $12 \mathrm{mMol} / \mathrm{L}$ Adenosine diphosphate, $60 \mathrm{mMol} / \mathrm{L}$ each of $\mathrm{D}$-glucose and $\mathrm{Mg}$ acetate, $6 \mathrm{mMol} / \mathrm{L}$ nicotinamide adenine dinucleotide, $9000 \mathrm{U} / \mathrm{L}$ of Hexokinase and $7500 \mathrm{U} / \mathrm{L}$ of glucose-6-phosphate dehydrogenase in $100 \mathrm{mMol} / \mathrm{L} \mathrm{pH}$ 7.6 Tris buffer. The AK activity on the gel was visualized using the characteristic $360 \mathrm{~nm}$ fluorescence of the NADH product formed at the site of isoenzyme activity, based on the coupled enzyme reaction scheme shown below:

$$
\begin{aligned}
& \text { Adenylate kinase } \\
& 2 \mathrm{ADP} \longrightarrow \mathrm{ATP}+\mathrm{AMP}
\end{aligned}
$$

Hexokinase

$$
\text { ATP + D-Glucose } \longrightarrow \text { D-Glucose-6-phosphate + ADP }
$$

G-6-P dehydrogenase

D-Glu-6-PO4 + NAD $\longrightarrow$ 6-phosphogluconate + NADH

Total AK activity in serum was measured using a Cobasfara centrifugal analyzer (Roche Diagnostics, Somerville, NJ) employing a 10-fold diluted AK visualization reagent described above. The diluted reagent is comparable to that described by Brolin (2). In this assay, performed in a centrifugal analyzer at $37^{\circ} \mathrm{C}, 5 \mu \mathrm{l}$ sample is mixed with $250 \mu \mathrm{l}$ of the diluted $\mathrm{AK}$ reagent containing $1.2 \mathrm{mMol} / \mathrm{L}$ adenosine diphosphate (ADP); $6 \mathrm{mMol} / \mathrm{L}$ each of glucose, magnesium acetate and nicotinamide adenine dinucleotide (NAD); 9 units of hexokinase and 7.5 units of glucose-6-phosphate dehydrogenase. The absorbance of NADH is measured at $360 \mathrm{~nm}$, every $15 \mathrm{sec}$ interval for the next $5 \mathrm{~min}$, and the AK activity in

\footnotetext{
${ }^{1}$ Summer undergraduate research student.

*Correspondence to: Vadiraja V. Murthy, Special Chemistry Laboratories, Room 2 S-11, Nurses Residence Building, Jacobi Medical Center, Bronx, New York 10461.
}

Received 3 March 1997; Accepted 4 April 1997 
$\mathrm{U} / \mathrm{L}$ is computed from the rate of change in absorbance/min and the known molar absorptivity of NADH at $360 \mathrm{~nm}$.

For measurement of erythrocyte AK activity we analyzed the sample by agarose gel electrophoresis in Tris-barbital buffer using the Helena REP reagents and procedure. Percent erythrocyte $\mathrm{AK}$ isoenzyme was computed by scanning the NADH fluorescence associated with the isoenzyme activity spot on the gel. The erythrocyte AK isoenzyme activity was computed by multiplying \% erythrocyte AK isoenzyme fraction with the total activity of the sample in U/L.

The extent of hemolysis was estimated by measuring serum hemoglobin concentration in a Coulter STKS instrument (Coulter Corp., Hialeah, FL).

\section{CLINICAL SAMPLES}

In a preliminary experiment, random serum samples from 20 hospitalized patients were analyzed. Of these 15 samples exhibited various degrees of red pigmentation on visual inspection. The remaining 5 serum samples did not show any visual hemolysis.

As part of an ongoing investigation, we also analyzed serum samples from 20 (9 males and 11 females ranging in ages between 19 and 64) anemic hospitalized patients (group I) consisting of 18 sickle cell anemia patients, one patient with thrombotic thrombocytopenic purpura (TTP) and another patient with delayed transfusion reaction along with samples from a matched group (according to sex and age range) of 20 suspected MI patients (group II) and samples from 20 healthy blood donors of ages 18-45 (group III).

We also measured AK enzyme activities in both plasma and serum samples obtained from 5 male and 5 female sickle cell anemia patients between ages 21 and 56 .

\section{Sample Preparation}

All serum samples were prepared by collecting blood in speckled red top vacutainer tubes. After allowing $30 \mathrm{~min}$ for the blood to clot, the samples were centrifuged for $5 \mathrm{~min}$ at $1500 \times \mathrm{g}$ in a clinical centrifuge. Plasma samples were prepared from blood samples collected in green top vacutainer tubes containing sodium heparin. After inverting the tubes gently 8 times to prevent clotting, the samples were centrifuged as described above to recover plasma.

The clinical diagnosis of hemolysis in anemic hospitalized patients was made using an elevation of bilirubin concentra-

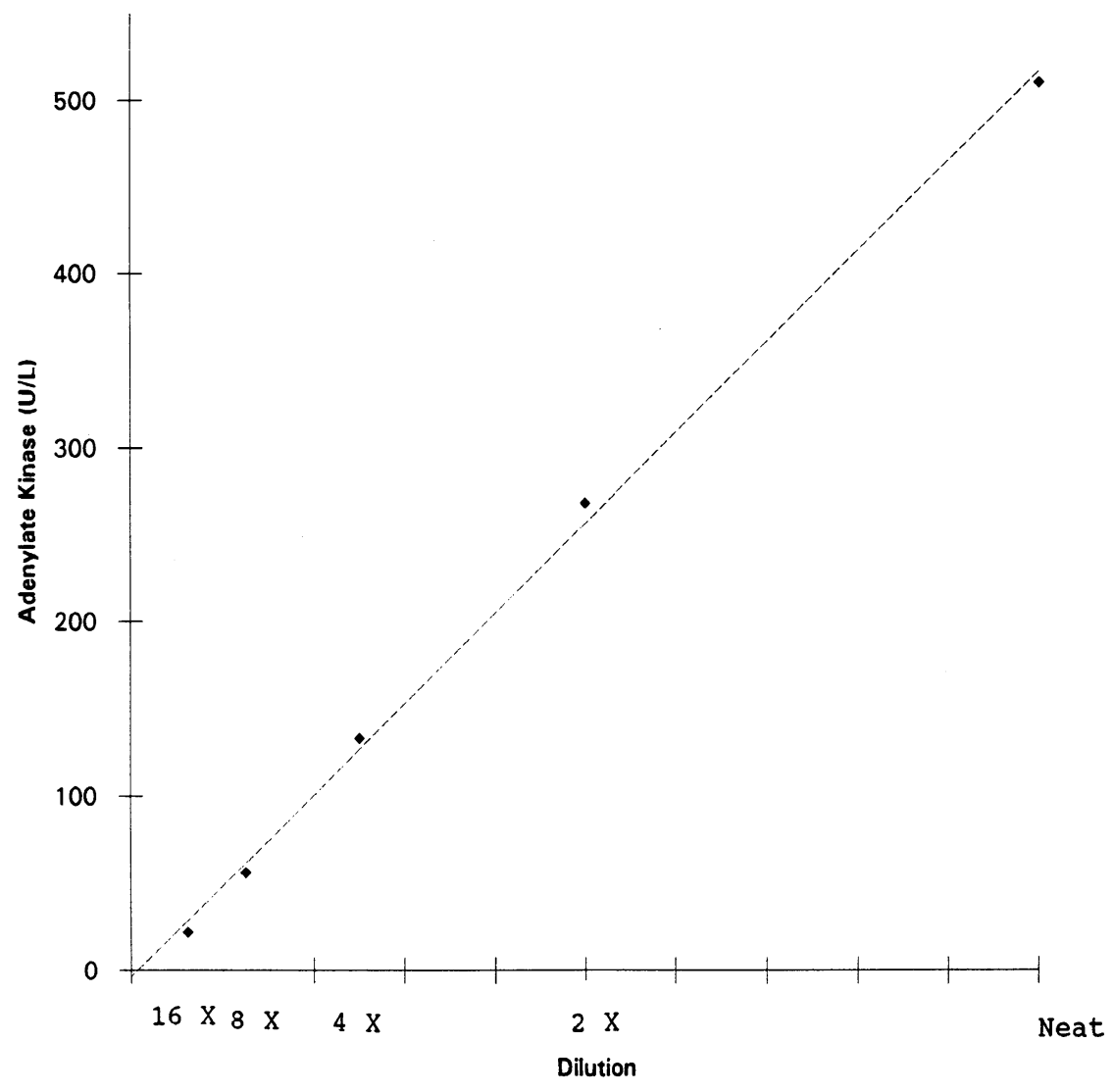

Fig. 1. Linearity of adenylate kinase assay. A freshly prepared red blood cell hemolysate containing high erythrocyte adenylate kinase (AK) activity was serially diluted with a solution of $9 \mathrm{~g} / \mathrm{L} \mathrm{NaCl}$ (sa-

line) and the diluted samples were assayed for AK activity with the Cobas-fara centrifugal analyzer. 
TABLE 1. Effect of Hemolysis on Adenylate Kinase Activity in Serum

\begin{tabular}{|c|c|c|c|c|c|}
\hline \multirow[b]{2}{*}{ Sample No. ${ }^{a}$} & \multirow[b]{2}{*}{ Hemoglobin $(\mathrm{g} / \mathrm{L})$} & \multicolumn{2}{|c|}{ Adenylate kinase (U/L) } & \multicolumn{2}{|c|}{$\begin{array}{l}\text { Average Erythrocyte } \\
\text { AK isoenzyme }\end{array}$} \\
\hline & & Run I & Run II & $(\%)$ & $(\mathrm{U} / \mathrm{L})$ \\
\hline 1 & 1.0 & 21 & 22 & 100 & 22 \\
\hline 2 & 1.0 & 48 & 48 & 98 & 47 \\
\hline 3 & 1.0 & 21 & 21 & 100 & 21 \\
\hline 4 & 2.0 & 45 & 44 & 99 & 44 \\
\hline 5 & 2.0 & 48 & 48 & 98 & 47 \\
\hline 6 & 2.0 & 58 & 56 & 99 & 55 \\
\hline 7 & 2.0 & 53 & 53 & 100 & 53 \\
\hline 8 & 2.0 & 64 & 63 & 100 & 64 \\
\hline 9 & 2.0 & 88 & 86 & 99 & 85 \\
\hline 10 & 2.0 & 157 & 150 & 98 & 148 \\
\hline 11 & 3.0 & 82 & 85 & 99 & 84 \\
\hline 12 & 3.0 & 172 & 165 & 99 & 167 \\
\hline 13 & 5.0 & 153 & 147 & 98 & 145 \\
\hline 14 & 5.0 & 472 & 454 & 99 & 454 \\
\hline 15 & 6.0 & 194 & 187 & 100 & 190 \\
\hline 16 & 0 & 1 & 0 & 0 & 0 \\
\hline 17 & 0 & 5 & 5 & 0 & 0 \\
\hline 18 & 0 & 5 & 5 & 0 & 0 \\
\hline 19 & 0 & 11 & 11 & 0 & 0 \\
\hline 20 & 0 & 32 & 32 & 0 & 0 \\
\hline
\end{tabular}

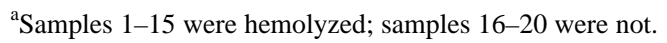

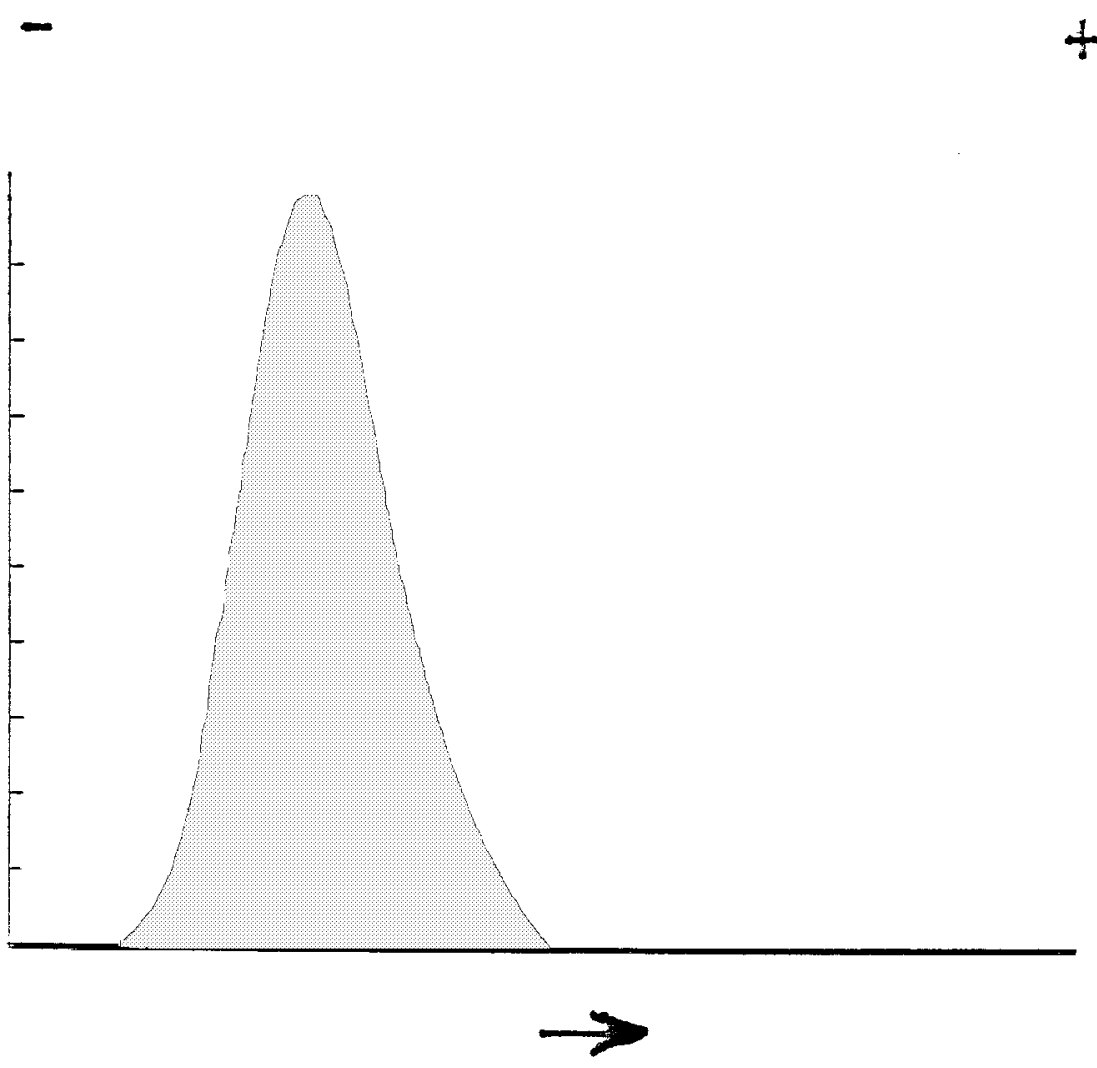

Fig. 2. Erythrocyte AK isoenzyme activity. Scan representing AK isoenzyme activity, after electrophoretic fractionation of a freshly prepared red blood cell hemolysate on agarose gel with the Helena REP instrument. Anode is to the right and the migration is from left to right. The fluorescent band migrating near the cathode represents erythrocyte AK isoenzyme activity. 


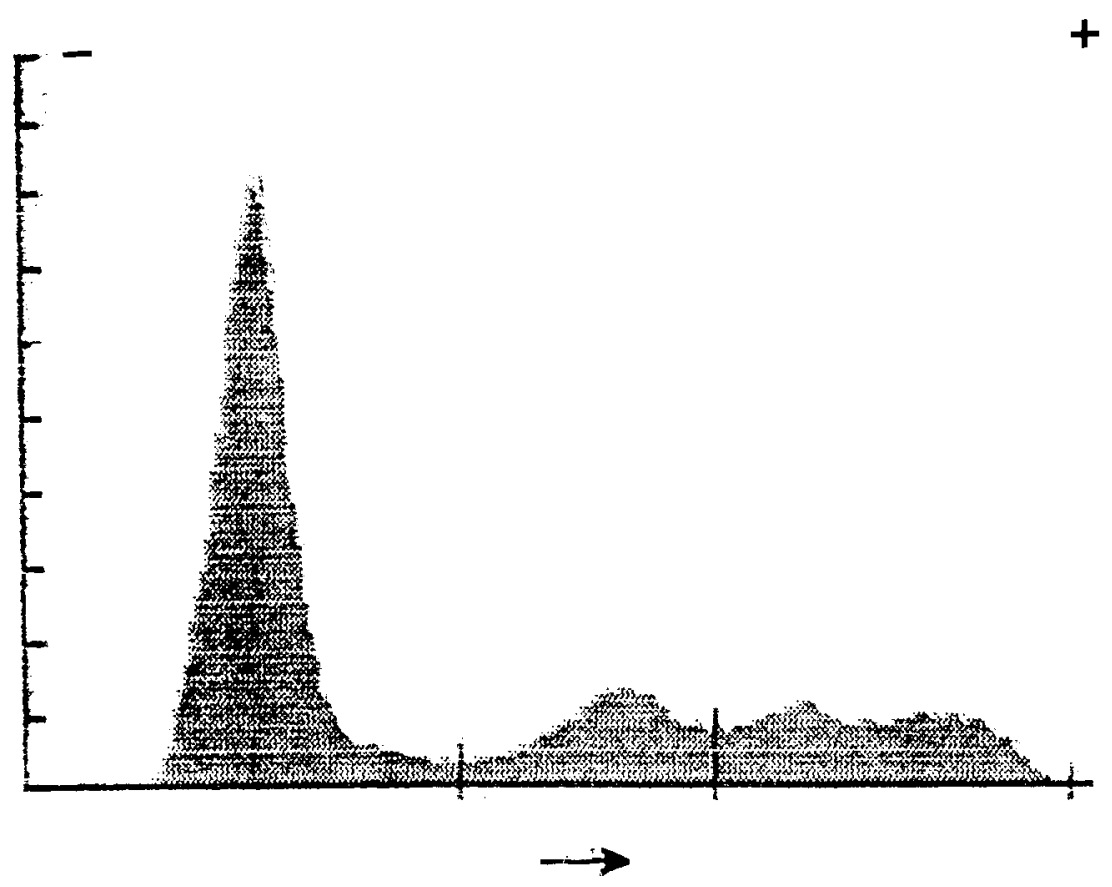

Fig. 3. Scan of AK isoenzyme distribution pattern following electrophoretic fractionation of a hemolyzed serum sample. A hemolyzed serum sample, containing $2 \mathrm{~g} / \mathrm{L}$ hemoglobin and exhibiting $87 \mathrm{U} / \mathrm{L}$ of AK activity, was subjected to $\mathrm{AK}$ isoenzyme electrophoretic fractionation on agarose gel. The slow moving fluorescent band migrating near the cathode represents the major erythrocyte AK isoenzyme component (62\%) in hemolyzed serum. See Fig. 2 for other details.

were used as the source of AK enzyme activity (Figure 1). The total AK activity in the serum samples analyzed in this study, ranged from 5-464 U/L as indicated in Table 1. Results of duplicate assays of serum samples agreed well. The serum samples with measurable total AK activity were subjected to electrophoresis in the REP CK instrument, employing agarose gels routinely used for CK fractionation, and visualized with AK visualization reagent. A typical scan depicting the mobility of erythrocyte AK using a freshly prepared red blood cell hemolysate is shown in Figure 2. A single band of AK activity migrating near the cathode was seen indicating that all the AK activity in the cell-free supernatant of erythrocytes is attributable to a single AK isoenzyme.

Similarly, red tinged serum samples with measurable total AK activity were subjected to electrophoresis in the REP in-

TABLE 2. Comparison of Erythrocyte Adenylate Kinase Activity in Serum Samples from Patients With and Without Hemolytic Anemia

\begin{tabular}{lcc}
\hline Patient category (n) & Mean plasma Hb (g/dL) & $\begin{array}{c}\text { Erythroctye AK isoenzyme (U/L) } \\
\text { Mean Activity } \pm \text { S.D. (Range) }\end{array}$ \\
\hline $\begin{array}{l}\text { Group I (20) } \\
\quad \text { hemolytic anemia patients) }\end{array}$ & $<0.1^{\mathrm{A}}$ & $48 \pm 57$ \\
$\begin{array}{l}\text { Group II (20) } \\
\quad \text { (non hemolytic patients) }\end{array}$ & $<0.1$ & $0.1 \pm 0.3$ \\
$\begin{array}{l}\text { Group III (20) } \\
\quad \text { Blood donors) }\end{array}$ & $<0.1$ & $0.0 \pm 0.4$ \\
\hline
\end{tabular}

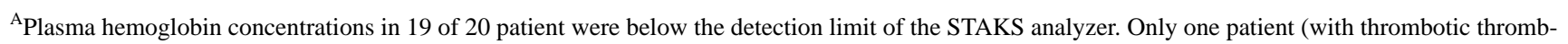
ocytopenic purpura) in this group exhibited visible hemolysis with a plasma hemogloin concentration of $2.3 \mathrm{~g} / \mathrm{dL}$. 
TABLE 3. Adenylate Kinase Activity in Serum and Plasma of Hemolytic Anemia patients

\begin{tabular}{lcc}
\hline & \multicolumn{2}{c}{ Adenylate kinase (U/L) } \\
\cline { 2 - 3 } Patient No. & Serum & Plasma $^{\mathrm{A}}$ \\
\hline 1 & 28 & 29 \\
2 & 15 & 14 \\
3 & 36 & 37 \\
4 & 25 & 24 \\
5 & 173 & 176 \\
6 & 203 & 206 \\
7 & 29 & 29 \\
8 & 68 & 69 \\
9 & 61 & 62 \\
10 & 119 & 121 \\
\hline
\end{tabular}

${ }^{\text {A }}$ Plasma samples were prepared from heparinized blood samples from the same patients.

strument and the gel was developed with AK reagent to visualize the fluorescent isoenzyme activity under UV light. All 15 serum samples with obvious hemolysis, exhibited AK isoenzyme activity migrating close to the cathode and behaving like CK-MM after electrophoresis (1). A typical scan of AK isoenzyme fractionation is shown in Figure 3. Majority of the AK activity present in hemolyzed serum samples consisted of erythrocyte $\mathrm{AK}$ isoenzyme activity giving a mean \pm S.D. activity of $99.0 \pm 0.8 \%$ (from Table 1 ). This unique AK isoenzyme activity must be originating from the erythrocyte following in vivo or in vitro hemolysis was further confirmed by the characteristic absence of a similar fluorescent band of AK isoenzyme activity in all 5 of the non-hemolyzed serum samples examined with our electrophoresis assay.

As part of our ongoing studies to confirm erythrocyte AK isoenzyme activity as a possible marker of hemolysis, we have recently analyzed serum samples from hospitalized anemic patients and compared them with a matching control group of non-anemic patients and a healthy donor group (Table 2). Serum samples from patients with confirmed hemolytic anemia (group I) exhibited significantly higher overall erythrocyte AK isoenzyme activity ranging from 4-206 U/L with a mean \pm S.D. of $48 \pm 57 \mathrm{U} / \mathrm{L}$. In contrast, serum samples from non-anemic patients (group II) exhibited no isoenzyme activity with the exception of one sample which showed a low activity of $0.8 \mathrm{U} / \mathrm{L}$. Similarly the samples from normal blood donors (group III) exhibited no erythrocyte AK isoenzyme activity in 19 samples and a very low activity of $0.4 \mathrm{U} / \mathrm{L}$ in one case. The results of AK enzyme assays using either serum or plasma sample from the same patient were very similar as shown in Table 3.

\section{DISCUSSION}

Adenylate kinase isoenzyme originating from erythrocyte has been shown to give falsely elevated CK and CK isoenzyme assay results when serum samples are hemolyzed (1). A simple quantitative test for assessing hemolysis was not available until now. The ability to detect and quantify AK isoenzyme activity present in erythrocyte can be effectively used for the purpose of identifying hospitalized anemic patients susceptible to hemolysis from a variety of causes like drug induced hemolysis, fragility and loss of red cell membrane permeability or mismatched transfusion.

Serum samples from patients diagnosed with hemolytic anemia exhibited increased erythrocyte AK isoenzyme activity in serum while those from patients without any signs of hemolytic anemia showed little or no erythrocyte AK isoenzyme activity with our electrophoresis assay. We have presented preliminary evidence for identifying hemolytic anemia patients earlier by determining erythrocyte AK isoenzyme activity in serum (or plasma) rather than using measurement of plasma hemoglobin concentration.

Plasma samples may also be used for measuring AK activ-

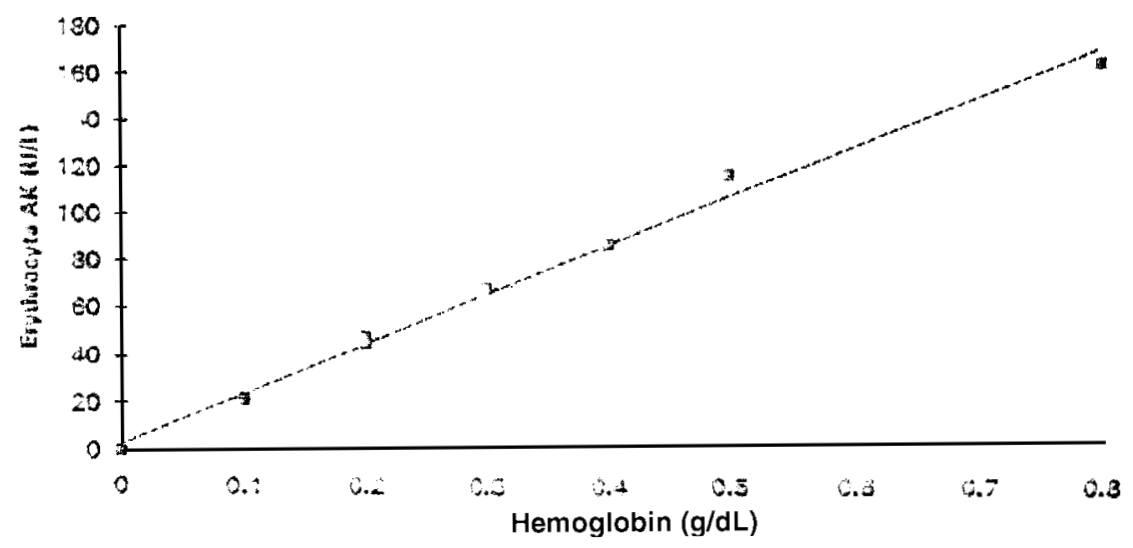

Fig. 4. Correlation between erythrocyte AK isoenzyme activity and hemoglobin concentration in hemolyzed serum samples. Erythrocyte AK isoenzyme activity and hemoglobin concentration in hemolyzed serum samples. Erythrocyte AK isoenzyme activity in red tinged serum samples were quan- tified by multiplying the total AK activity and the \% erythrocyte $\mathrm{AK}$ isoenzyme as determined with the AK isoenzyme assay. The same samples were also analyzed for hemoglobin content with the Coulter STKS analyzer as described in the text. 
ity since AK assay results of both plasma and serum samples were similar (Table 3 ).

The current practice for laboratory diagnosis of hemolysis or red blood cell destruction, based on a combination of elevated serum LD activity and bilirubin coupled with elevated reticulocyte count, is far from satisfactory. Serum LD concentration may also be elevated in hepatic, cardiac, pulmonary and placental diseases thus reducing its specificity for detecting hemolysis (3-5). Similarly, bilirubin concentration in serum may be elevated in intra- and extra-hepatic biliary disease and in severe hepatocellular dysfunction (6). Patients suspected of having hemolytic anemia also have cardiac and/ or liver disease(s) making the diagnosis of hemolytic anemia difficult, if not, impossible. A red cell specific marker like the erythrocyte AK isoenzyme, whose concentration would be elevated only in serum (or plasma), primarily in hemolytic states, would aptly fulfill the need for a specific test for early detection of hemolysis. Our results of LD assays showed considerable overlap between hemolytic anemia patients and nonhemolytic patients. The same trend was seen when serum bilirubin concentration or reticulocyte counts were used for comparison (7).

A report on the leakage of AK from stored blood cells by Olsson et al. (8) supports our approach of harnessing erythrocyte $\mathrm{AK}$ isoenzyme as a specific and sensitive marker for hemolysis. However, the use of total AK activity in serum is not recommended for the purpose, since AK isoenzymes originating from other sources like muscle (myokinase) and intestine may be present in addition to erythrocyte isoenzyme. The data presented in Table 1 clearly demonstrate that the total AK enzyme activity is not proportional to the hemoglobin concentration in hemolyzed serum, since the total AK activity in serum may also consist of AK isoenzyme activities arising from other sources besides erythrocytes.

Erythrocyte AK activity in serum persists for a long time and the isoenzyme activity in serum samples stored at $10^{\circ} \mathrm{C}$ did not change appreciably over a 1 month period. The persistence of erythrocyte AK isoenzyme activity, its relative in vitro stability in serum combined with greater analytical sensitivity, makes it an ideal candidate as an indicator of hemolysis. In those hemolyzed samples where hemoglobin concentration could be measured with certainty with the STKS analyzer, the AK isoenzyme activity correlated well with the hemoglobin concentration (Figure 4). The superior analytical sensitivity of AK isoenzyme activity assay enabled us to detect even marginal hemolysis much better when direct hemoglobin measurement was not possible due to the limitation of the assay in measuring low hemoglobin concentration (Table 2). In all patients with hemolytic anemia, the erythrocyte adenylate kinase activity was considerably higher than that found in patients without hemolytic anemia (group II) or in healthy donor subjects (group III in Table 2). The low levels of AK isoenzyme activity in the sera of 2 subjects, one each in groups II and III, might reflect minor degree of in vitro hemolysis during transport and processing of blood samples rather than the result of any in vivo hemolytic activity.

\section{Acknowledgments}

The authors thank Dr. Anjali Kale, M.D. for providing the clinical samples.

\section{REFERENCES}

1. Murthy VV: Adenylate kinase mimics creatine kinase-MM isoenzyme in a CK isoenzyme electrophoresis assay. J Clin LabAnal 8:140-143, 1994.

2. Brolin SE: Adenylate kinase (myokinase-ATP; AMP phosphotransferase, EC 2.7.4.3). In Methods of Enzymatic Analysis. Vol. III. Bergmeyer HU, ed. Verlag-Chemie, Weinheim, Germany 1983, pp 540-545.

3. Wolf PL: Interpretation of lactate dehydrogenase isoenzymes. Clin Lab Med 6:541-545, 1986.

4. Vassault A, Wahlefeld AW, Denke U: Lactate Dehydrogenase. In Methods of Enzymatic Analysis, 3rd edition, Vol. III. Bergmeyer, HU, Bergmeyer J, Grassl M, eds. Verlag-Chemie, Weinheim, Germany, 1983, pp 118-138.

5. Wilkinson JH: Lactate dehydrogenase. In The Principles and Practice of Diagnostic Enzymology. Edward Arnold, London, 1976.

6. Scharschmidt BF, Blanckaert N, Farina FA, Kabra PM, Stafford BE, Weisger RA: Measurement of bilirubin and its mono- and diconjugates: Application to patients with hepatobiliary disease. Gut, 23:643-649, 1982.

7. Kale A, Murthy VV, Burns ER. Clinical utility of serum erythrocyte adenylate kinase, a new marker for hemolysis. Blood 88:5b, 1996.

8. Olsson T, Gullicksson H, Palemborn M, Bergstrom K, Thore A: Leakage of adenylate kinase from stored blood cells. J Appl Biochem 5:437-445, 1983. 